\title{
A Discrete-Time Unreliable Geo/G/1 Retrial Queue with Balking Customers, Second Optional Service, and General Retrial Times
}

\author{
Feng Zhang and Zhifeng Zhu \\ College of Science, North University of China, Taiyuan 030051, China \\ Correspondence should be addressed to Feng Zhang; gigigi69@163.com
}

Received 12 January 2013; Revised 20 October 2013; Accepted 20 October 2013

Academic Editor: Yi-Kuei Lin

Copyright (c) 2013 F. Zhang and Z. Zhu. This is an open access article distributed under the Creative Commons Attribution License, which permits unrestricted use, distribution, and reproduction in any medium, provided the original work is properly cited.

\begin{abstract}
This paper deals with the steady-state behavior of a discrete-time unreliable Geo/G/1 retrial queueing system with balking customers and second optional service. The server may break down randomly while serving the customers. If the server breaks down, the server is sent to be repaired immediately. We analyze the Markov chain underlying the considered system and its ergodicity condition. Then, we obtain some performance measures based on the generating functions. Moreover, a stochastic decomposition result of the system size is investigated. Finally, some numerical examples are provided to illustrate the effect of some parameters on main performance measures of the system.
\end{abstract}

\section{Introduction}

During the past few decades, there has been increasing interest in studying retrial queueing systems because they are widely used in performance analysis of many practical systems such as call center, computer systems, and telecommunication networks. Retrial queueing systems are characterized by the feature that a blocked customer (a customer who finds the server unavailable) may leave the service area temporarily and join a retrial group in order to retry his request after some random time. For more detailed review of the main results and references on the retrial queueing systems, the readers are referred to [1-3].

In the past, the research of retrial queueing systems focused mainly on the continuous-time case $[4,5]$. Compared with the continuous-time counterparts, the discrete-time retrial queueing systems received less attention in the literature. One of the difficulties in developing the discrete-time retrial queueing systems lied in the fact that the complexity of the discrete-time retrial queueing models increases. The reason is that in a discrete-time retrial queueing model, arrivals, departures, and retrials can occur simultaneously; that is, the probability that two or more events occur at every slot is positive. In fact; the research on discrete-time retrial queueing systems is very important due to the fact that they are more appropriate than their continuous-time counterparts for modeling slotted digital computer and communication systems such as broadband integrated services digital network (B-ISDN) and time-division multiple access (TDMA) systems. The first work on discrete-time retrial queueing system was discussed by Yang and Li [6]. They extended the continuous-time $M / G / 1$ retrial queueing system to the discrete-time case and save the relationship between the $G e o / G / 1$ retrial queues and the corresponding continuous-time counterpart. Atencia and Moreno [7] generalized the model of Yang and Li to the discrete-time Geo/G/1 retrial queue with general retrial times. For related literature on discrete-time retrial queueing systems, the reader may refer to $[8,9]$.

Most of the retrial queueing models in the literature assumed that customers received service eventually. However, in practice, customers may balk or abandon the system before receiving service. Queueing models with balking or impatience customers arise in many diverse applications. Although the incorporation of balking or impatience customers in retrial queueing systems is very important, so far there is only little work on retrial queueing systems with balking or impatience customers in the literature [10-12]. On the other hand, queueing models in which the server may provide a second phase service have been proved very useful to analyze many practical situations, arising in manufacturing systems, call center, and computer systems. The retrial queueing systems with second phase service were studied by 
Artalejo and Choudhury [13], Atencia and Moreno [14], and Choudhury and Deka [15].

In many practical situations, the server may be subjected to unpredictable breakdowns. The breakdowns of the server may have a great influence on performance measures of some practical systems. So, the research of retrial queues with unreliable server is very important. There have been significant contributions to the retrial queueing systems with unreliable server. For example, the continuous-time retrial queueing systems which take into account servers breakdowns and repairs were discussed by Kulkarni and Choi [16], Wang et al. $[17,18]$, and Li et al. [19]. Parallel to the continuous-time unreliable retrial queues, the discrete-time retrial queues with starting failure were studied by Atencia and Moreno [20], Wang and Zhao [21], and Atencia et al. [22]. The discretetime unreliable retrial queueing systems caused by negative customers were discussed by Wang and Zhang [23]. In contrast to the retrial queueing system with starting failure, the analysis of discrete-time retrial queueing system is more complex. Though the discrete-time retrial queueing systems with unreliable server have been studied by some authors, there is little work about the discrete-time unreliable retrial queueing systems which assumed that the server's life is random time [24].

To the best of our knowledge, there is no published work on discrete-time $G e o / G / 1$ retrial queueing system with balking customer, second optional service, and unreliable server. The main objective in this paper is to extend the analysis of continuous-time $M / G / 1$ retrial queueing system with second optional service [13], exponential server lifetime [17], and general retrial times to discrete-time case. Moreover, our model generalized the discrete-time retrial queueing system studied by Atencia and Moreno [7] and Aboul-Hassan et al. [11] directly. Our queueing system can be used to model many practical situations. For example, in the Voice-over-IP (VoIP) system, the analogue voice information is digitized, sent to the destination, and converted to the original analogue voice signal. In the existing VoIP system, the admission control is proposed for improving the quality of service (QoS) and the VoIP system may reject some data packets request due to network congestion. The admitted packets can be placed in the buffer and then can be sent to another end user for further process. The VoIP systems may break down during establishing connection, digitizing, and transferring information. As soon as the VoIP system breaks down, it is repaired immediately.

The rest of the paper is organized as follows. In Section 2, we give a detailed description of the mathematical model. In Section 3, the Markov chain underlying the queueing system is analyzed and some performance measures of the system are also obtained. In Section 4, we derive a stochastic decomposition result. Finally, some numerical results are provided to show the impact of the some parameters on the performance measures of the system in Section 5.

\section{The Mathematical Model}

We consider a discrete-time single server retrial queue where the time axis is segmented into slots of equal length and all queueing activities occur at the slot boundaries. Let the time axis be marked by $0,1,2, \ldots, m, \ldots$. Specially, we assume that the departures and the end of the repairs occur in the interval $\left(m^{-}, m\right)$, and the arrivals, the retrials, and the beginning of the repairs occur in the interval $\left(m, m^{+}\right)$. That is, we consider an early arrival system (EAS) policy.

Customers arrive at the system according to a geometric arrival process with parameter $p$, where $p$ is the probability that an arrival occurs in a slot. If an arriving customer finds that the server is idle, he begins his service immediately and leaves the system forever after service completion. Otherwise, if an arriving customer finds that the server is busy or under repair, he joins the orbit with probability $\alpha$ or leaves the system completely with probability $1-\alpha$. We assumed that only the first customer in the orbit is permitted to access to the server. Successive interretrial times of any customer follow an arbitrary distribution $\left\{a_{i}\right\}_{i=0}^{\infty}$ with generating function $A(x)=$ $\sum_{i=0}^{\infty} a_{i} x^{i}$.

The server provides two phases of different services for customers in succession. As soon as the first-phase service (FPS) of a customer is completed, he may receive the secondphase service (SPS) with probability $r$ or leaves the system with probability $\bar{r}=1-r$. The service times of the FPS and SPS are independent and arbitrarily distributed with distributions $\left\{s_{1, i}\right\}_{i=0}^{\infty},\left\{s_{2, i}\right\}_{i=0}^{\infty}$, and generating functions $S_{1}(x)=\sum_{i=0}^{\infty} s_{1, i} x^{i}$, $S_{2}(x)=\sum_{i=0}^{\infty} s_{2, i} x^{i}$, respectively. The corresponding $n$th factorial moments are $\beta_{1, n}$ and $\beta_{2, n}$, respectively.

The server may break down during serving customers. If the server breaks down, it is sent to repair immediately; the customer just being served before the server breakdown waits in the service station until the server is repaired to complete his remaining service. It is assumed that the lifetimes of the server for the FPS and SPS are geometrically distributed with parameters $\bar{\theta}_{1}=1-\theta_{1}$ and $\bar{\theta}_{2}=1-\theta_{2}$, respectively, where $\theta_{1}$ and $\theta_{2}$ are the probability that the failures do not occur in a slot, respectively.

The repair times for the FPS and SPS are independent and identically distributed with arbitrary distributions $\left\{s_{3, i}\right\}_{i=0}^{\infty}$ and $\left\{s_{4, i}\right\}_{i=0}^{\infty}$, respectively. The corresponding generating functions and $n$th factorial moments are $S_{3}(x)=\sum_{i=0}^{\infty} s_{3, i} x^{i}$, $S_{4}(x)=\sum_{i=0}^{\infty} s_{4, i} x^{i}, \beta_{3, n}$ and $\beta_{4, n}$, respectively.

Finally, we suppose that various stochastic processes involved in the system are independent of each other. In order to avoid trivial cases, it is also supposed that $0<p<1$, $0 \leq r<1,0<\theta_{1} \leq 1,0<\theta_{2} \leq 1$.

\section{The Markov Chain}

At time $m^{+}$, the system can be described by the process $Y_{m}=\left(C_{m}, \xi_{0, m}, \xi_{1, m}, \xi_{2, m}, \xi_{3, m}, \xi_{4, m}, N_{m}\right)$, where $C_{m}$ denotes the state of the server, $0,1,2,3$, or 4 , according to whether the server is free, busy with FPS, busy with SPS, under repair during FPS, or under repair during SPS, and $N_{m}$ is the number of the customers in the orbit. If $C_{m}=0$, then $\xi_{0, m}$ represents the remaining retrial time. If $C_{m}=1,2$, then $\xi_{1, m}, \xi_{2, m}$ represent the customers' remaining service time for FPS and SPS, respectively. If $C_{m}=3,4$, then $\xi_{1, m}, \xi_{2, m}$ represent the remaining service time of the customer who was 
being served just before the server breakdown and $\xi_{3, m}, \xi_{4, m}$ represent the remaining repair time of the server for the FPS and SPS, respectively. It can be shown that $\left\{Y_{m}, m \geq 1\right\}$ is a Markov chain with the following state space:

$$
\begin{aligned}
\Omega & =\{(0,0)\} \cup\{(0, i, k): i \geq 1, k \geq 1\} \\
& \cup\{(1, i, k): i \geq 1, k \geq 0\} \cup\{(2, i, k): i \geq 1, k \geq 0\} \\
& \cup\{(3, i, j, k): i \geq 1, j \geq 1, k \geq 0\} \\
& \cup\{(4, i, j, k): i \geq 1, j \geq 1, k \geq 0\} .
\end{aligned}
$$

Define the stationary probabilities of the Markov chain as follows:

$$
\begin{gathered}
\pi_{0,0}=\lim _{m \rightarrow \infty} P\left\{C_{m}=0, N_{m}=0\right\}, \\
\pi_{0, i, k}=\lim _{m \rightarrow \infty} P\left\{C_{m}=0, \xi_{0, m}=i, N_{m}=k\right\} ; \quad i \geq 1, k \geq 1, \\
\pi_{n, i, k}=\lim _{m \rightarrow \infty} P\left\{C_{m}=n, \xi_{1, m}=i, N_{m}=k\right\} ; \\
n=1,2, i \geq 1, k \geq 0, \\
\pi_{n, i, j, k}=\lim _{m \rightarrow \infty} P\left\{C_{m}=n, \xi_{1, m}=i, \xi_{3, m}=j, N_{m}=k\right\} ; \\
n=3,4, i \geq 1, j \geq 1, k \geq 0 .
\end{gathered}
$$

Then, the Kolmogorov equations for the stationary distribution are

$$
\begin{aligned}
\pi_{0,0}=\bar{p} \pi_{0,0}+\bar{p} \bar{r} \pi_{1,1,0}+\bar{p} \pi_{2,1,0} \\
\pi_{0, i, k}=\bar{p} \pi_{0, i+1, k}+\bar{p} a_{i} \bar{r} \pi_{1,1, k}+\bar{p} a_{i} \pi_{2,1, k}, \quad i \geq 1, k \geq 1 \\
\pi_{1, i, k}=\delta_{0, k} p \theta_{1} s_{1, i} \pi_{0,0}+\left(1-\delta_{0, k}\right) p \theta_{1} s_{1, i} \sum_{j=1}^{\infty} \pi_{0, j, k} \\
+\bar{p} \theta_{1} s_{1, i} \pi_{0,1, k+1}+p \theta_{1} s_{1, i} \bar{r} \pi_{1,1, k} \\
+\bar{p} a_{0} \theta_{1} \bar{r} s_{1, i} \pi_{1,1, k+1}+p \theta_{1} s_{1, i} \pi_{2,1, k} \\
+\bar{p} a_{0} \theta_{1} s_{1, i} \pi_{2,1, k+1}+(\bar{p}+p \bar{\alpha}) \theta_{1} \pi_{1, i+1, k} \\
+\left(1-\delta_{0, k}\right) p \alpha \theta_{1} \pi_{1, i+1, k-1}+\left(1-\delta_{0, k}\right) p \alpha \theta_{1} \pi_{3, i, 1, k-1} \\
+(\bar{p}+p \bar{\alpha}) \theta_{1} \pi_{3, i, 1, k}, \quad i \geq 1, k \geq 0, \\
\pi_{2, i, k}=(\bar{p}+p \bar{\alpha}) r \theta_{2} s_{2, i} \pi_{1,1, k}+\left(1-\delta_{0, k}\right) \\
+\times p \alpha r \theta_{2} s_{2, i} \pi_{1,1, k-1}+(\bar{p}+p \bar{\alpha}) \theta_{2} \pi_{2, i+1, k} \\
+\left(1-\delta_{0, k}\right) p \alpha \theta_{2} \pi_{2, i+1, k-1}+\left(1-\delta_{0, k}\right) p \alpha \theta_{2} \pi_{4, i, 1, k-1} \\
+(\bar{p}+p \bar{\alpha}) \theta_{2} \pi_{4, i, 1, k}, \quad i \geq 1, k \geq 0,
\end{aligned}
$$

$$
\begin{aligned}
& \pi_{3, i, j, k}=\delta_{0, k} p \bar{\theta}_{1} s_{1, i} s_{3, j} \pi_{0,0}+\left(1-\delta_{0, k}\right) \\
& \times p \bar{\theta}_{1} s_{1, i} s_{3, j} \sum_{l=1}^{\infty} \pi_{0, l, k}+\bar{p}_{1} s_{1, i} s_{3, j} \pi_{0,1, k+1} \\
& +p \bar{\theta}_{1} s_{1, i} s_{3, j} \bar{r} \pi_{1,1, k}+\bar{p} \bar{\theta}_{1} a_{0} s_{1, i} s_{3, j} \bar{r} \pi_{1,1, k+1} \\
& +p \bar{\theta}_{1} s_{1, i} s_{3, j} \pi_{2,1, k}+\bar{p}_{1} a_{0} s_{1, i} s_{3, j} \pi_{2,1, k+1} \\
& +\left(1-\delta_{0, k}\right) p \alpha \bar{\theta}_{1} s_{3, j} \pi_{1, i+1, k-1} \\
& +(\bar{p}+p \bar{\alpha}) \bar{\theta}_{1} s_{3, j} \pi_{1, i+1, k}\left(1-\delta_{0, k}\right) p \alpha \bar{\theta}_{1} s_{3, j} \pi_{3, i, 1, k-1} \\
& +(\bar{p}+p \bar{\alpha}) \bar{\theta}_{1} s_{3, j} \pi_{3, i, 1, k}+(\bar{p}+p \bar{\alpha}) \pi_{3, i, j+1, k} \\
& +\left(1-\delta_{0, k}\right) p \alpha \pi_{3, i, j+1, k-1}, \quad i \geq 1, j \geq 1, k \geq 0, \\
& \pi_{4, i, j, k}=(\bar{p}+p \bar{\alpha}) r \bar{\theta}_{2} s_{2, i} s_{4, j} \pi_{1,1, k} \\
& +\left(1-\delta_{0, k}\right) \operatorname{p\alpha r} \bar{\theta}_{2} s_{2, i} s_{4, j} \pi_{1,1, k-1} \\
& +\left(1-\delta_{0, k}\right) p \alpha \bar{\theta}_{2} s_{4, j} \pi_{2, i+1, k-1} \\
& +(\bar{p}+p \bar{\alpha}) \bar{\theta}_{2} s_{4, j} \pi_{2, i+1, k} \\
& +\left(1-\delta_{0, k}\right) p \alpha \bar{\theta}_{2} s_{4, j} \pi_{4, i, 1, k-1} \\
& +(\bar{p}+p \bar{\alpha}) \bar{\theta}_{2} s_{4, j} \pi_{4, i, 1, k} \\
& +(\bar{p}+p \bar{\alpha}) \pi_{4, i, j+1, k}+\left(1-\delta_{0, k}\right) p \alpha \pi_{4, i, j+1, k-1}, \\
& i \geq 1, \quad j \geq 1, \quad k \geq 0,
\end{aligned}
$$

with the normalizing condition

$$
\begin{aligned}
\pi_{0,0}+ & \sum_{i=1}^{\infty} \sum_{k=1}^{\infty} \pi_{0, i, k}+\sum_{n=1}^{2} \sum_{i=1}^{\infty} \sum_{k=0}^{\infty} \pi_{n, i, k} \\
& +\sum_{l=3}^{4} \sum_{i=1}^{\infty} \sum_{j=1}^{\infty} \sum_{k=0}^{\infty} \pi_{l, i, j, k}=1,
\end{aligned}
$$

where $\delta_{0, k}$ denotes the Kronecker delta. To resolve (3)-(8), we introduce the following generating functions:

$$
\begin{gathered}
\phi_{0}(x, z)=\sum_{i=1}^{\infty} \sum_{k=1}^{\infty} \pi_{0, i, k} x^{i} z^{k}, \\
\phi_{n}(x, z)=\sum_{i=1}^{\infty} \sum_{k=0}^{\infty} \pi_{n, i, k} x^{i} z^{k}, \quad n=1,2, \\
\phi_{l}(x, y, z)=\sum_{i=1}^{\infty} \sum_{j=1}^{\infty} \sum_{k=0}^{\infty} \pi_{l, i, j, k} x^{i} y^{j} z^{k}, \quad l=3,4,
\end{gathered}
$$


and the auxiliary generating functions:

$$
\begin{gathered}
\phi_{0, i}(z)=\sum_{k=1}^{\infty} \pi_{0, i, k} z^{k}, \quad i \geq 1, \\
\phi_{n, i}(z)=\sum_{k=0}^{\infty} \pi_{n, i, k} z^{k}, \quad n=1,2, \quad i \geq 1, \\
\phi_{l, i, j}(z)=\sum_{k=0}^{\infty} \pi_{l, i, j, k} z^{k}, \quad l=3,4, \quad i \geq 1, \quad j \geq 1, \\
\phi_{m, i, j}(y, z)=\sum_{k=0}^{\infty} \pi_{m, i, j, k} y^{j} z^{k}, \quad m=3,4, \quad i \geq 1, \quad j \geq 1, \\
\psi_{1}(x, z)=\sum_{i=1}^{\infty} \sum_{k=0}^{\infty} \pi_{3, i, 1, k} x^{i} z^{k} \\
\psi_{2}(x, z)=\sum_{i=1}^{\infty} \sum_{k=0}^{\infty} \pi_{4, i, 1, k} x^{i} z^{k}
\end{gathered}
$$

Multiplying (4)-(8) by $z^{k}$ and summing over $k$, we get the following equations:

$$
\begin{aligned}
\phi_{0, i}(z)= & \bar{p} \phi_{0, i+1}(z)+\bar{p} a_{i} \bar{r} \phi_{1,1}(z) \\
& +\bar{p} a_{i} \phi_{2,1}(z)-\bar{p} a_{i} \bar{r} \pi_{1,1,0}-\bar{p} a_{i} \pi_{2,1,0}, \quad i \geq 1 \\
\phi_{1, i}(z)= & p \theta_{1} s_{1, i} \pi_{0,0}+p \theta_{1} s_{1, i} \sum_{j=1}^{\infty} \phi_{0, j}(z) \\
& \bar{p}_{\theta_{1} s_{1, i} \phi_{0,1}(z)+p \theta_{1} s_{1, i} \bar{r} \phi_{1,1}(z)} \\
+ & \frac{\bar{p}}{z} \theta_{1} a_{0} s_{1, i} \bar{r}\left(\phi_{1,1}(z)-\pi_{1,1,0}\right)+p \theta_{1} s_{1, i} \phi_{2,1}(z) \\
& \bar{p} \theta_{1} a_{0} s_{1, i}\left(\phi_{2,1}(z)-\pi_{2,1,0}\right) \\
& +(\bar{p}+p \bar{\alpha}+z p \alpha) \theta_{1} \phi_{1, i+1}(z) \\
+ & (\bar{p}+p \bar{\alpha}+z p \alpha) \theta_{1} \phi_{3, i, 1}(z), \quad i \geq 1 \\
\phi_{3, i, j}(z)= & p \bar{\theta} s_{1, i} s_{3, j} \pi_{0,0}+p \bar{\theta}_{1} s_{1, i} s_{3, j} \sum_{l=1}^{\infty} \phi_{0, l}(z) \\
& +(\bar{p}+p \bar{\alpha}+z p \alpha) \theta_{2} \phi_{4, i, 1}(z) \\
& +(\bar{p}+p \bar{\alpha}+z p \alpha) \theta_{2} \phi_{2, i+1}(z) \\
\phi_{2, i}(z)= & (\bar{p}+p \bar{\alpha}+z p \alpha) \theta_{2} r s_{2, i} \phi_{1,1}(z) \\
&
\end{aligned}
$$

$$
\begin{aligned}
&+ \frac{\bar{p}}{z} \bar{\theta}_{1} a_{0} s_{1, i} s_{3, j} \bar{r}\left(\phi_{1,1}(z)-\pi_{1,1,0}\right) \\
&+ p \bar{\theta}_{1} s_{1, i} s_{3, j} \phi_{2,1}(z)+\frac{\bar{p}}{z} \bar{\theta}_{1} a_{0} s_{1, i} s_{3, j} \\
& \times\left(\phi_{2,1}(z)-\pi_{2,1,0}\right) \\
&+(\bar{p}+p \bar{\alpha}+z p \alpha) \bar{\theta}_{1} s_{3, j} \phi_{1, i+1}(z) \\
&+(\bar{p}+p \bar{\alpha}+z p \alpha) \bar{\theta}_{1} s_{3, j} \phi_{3, i, 1}(z) \\
&+(\bar{p}+p \bar{\alpha}+z p \alpha) \phi_{3, i, j+1}(z), \quad i \geq 1, \quad j \geq 1, \\
& \phi_{4, i, j}(z)=(\bar{p}+p \bar{\alpha}+z p \alpha) \bar{\theta}_{2} r s_{2, i} s_{4, j} \phi_{1,1}(z) \\
& \quad+(\bar{p}+p \bar{\alpha}+z p \alpha) \bar{\theta}_{2} s_{4, j} \phi_{2, i+1}(z) \\
& \quad+(\bar{p}+p \bar{\alpha}+z p \alpha) \bar{\theta}_{2} s_{4, j} \phi_{4, i, 1}(z) \\
& \quad+(\bar{p}+p \bar{\alpha}+z p \alpha) \phi_{4, i, j+1}(z), \quad i \geq 1 .
\end{aligned}
$$

By substituting (3) into (12), (13), and (15), we get

$$
\begin{aligned}
& \phi_{0, i}(z)=\bar{p} \phi_{0, i+1}(z)+\bar{p} a_{i} \bar{r} \phi_{1,1}(z) \\
& +\bar{p} a_{i} \phi_{2,1}(z)-\bar{p} a_{i} \pi_{0,0}, \quad i \geq 1, \\
& \phi_{1, i}(z)=\frac{\bar{p}}{z} \theta_{1} s_{1, i} \phi_{0,1}(z)+p \theta_{1} s_{1, i} \phi_{0}(1, z) \\
& +\left(p+\frac{\bar{p}}{z} a_{0}\right) \theta_{1} \bar{r} s_{1, i} \phi_{1,1}(z) \\
& +\left(p+\frac{\bar{p}}{z} a_{0}\right) \theta_{1} s_{1, i} \phi_{2,1}(z) \\
& +(\bar{p}+p \bar{\alpha}+z p \alpha) \theta_{1} \phi_{1, i+1}(z) \\
& +(\bar{p}+p \bar{\alpha}+z p \alpha) \theta_{1} \phi_{3, i, 1}(z) \\
& +\left(1-\frac{a_{0}}{z}\right) p \theta_{1} s_{1, i} \pi_{0,0}, \quad i \geq 1 \\
& \phi_{3, i, j}(z)=p \bar{\theta}_{1} s_{1, i} s_{3, j} \phi_{0}(1, z)+\frac{\bar{p}}{z} \bar{\theta}_{1} s_{1, i} s_{3, j} \phi_{0,1}(z) \\
& +\left(p+\frac{\bar{p}}{z} a_{0}\right) \bar{\theta}_{1} \bar{r} s_{1, i} s_{3, j} \phi_{1,1}(z) \\
& +\left(p+\frac{\bar{p}}{z} a_{0}\right) \bar{\theta}_{1} s_{1, i} s_{3, j} \phi_{2,1}(z) \\
& +(\bar{p}+p \bar{\alpha}+z p \alpha) \bar{\theta}_{1} s_{3, j} \phi_{1, i+1}(z) \\
& +(\bar{p}+p \bar{\alpha}+z p \alpha) \bar{\theta}_{1} s_{3, j} \phi_{3, i, 1}(z) \\
& +(\bar{p}+p \bar{\alpha}+z p \alpha) \phi_{3, i, j+1}(z) \\
& +\left(1-\frac{a_{0}}{z}\right) p \bar{\theta}_{1} s_{1, i} s_{3, j} \pi_{0,0}, \quad i \geq 1, j \geq 1 .
\end{aligned}
$$


Then, multiplying (16), (19) by $y^{j}$ and summing over $j$, we obtain

$$
\begin{aligned}
\phi_{4, i}(y, z)= & (\bar{p}+p \bar{\alpha}+z p \alpha) \bar{\theta}_{2} s_{2, i} S_{4}(y) r \phi_{1,1}(z) \\
& +(\bar{p}+p \bar{\alpha}+z p \alpha) \bar{\theta}_{2} S_{4}(y) \phi_{2, i+1}(z) \\
& +(\bar{p}+p \bar{\alpha}+z p \alpha)\left[\frac{\phi_{4, i}(y, z)}{y}-\phi_{4, i, 1}(z)\right] \\
& +(\bar{p}+p \bar{\alpha}+z p \alpha) \bar{\theta}_{2} S_{4}(y) \phi_{4, i, 1}(z), \\
& i \geq 1, \quad j \geq 1, \\
\phi_{3, i}(y, z)= & p \bar{\theta}_{1} s_{1, i} S_{3}(y) \phi_{0}(1, z)+\frac{\bar{p}}{z} \bar{\theta}_{1} s_{1, i} S_{3}(y) \phi_{0,1}(z) \\
& +\left(1-\frac{a_{0}}{z}\right) p \bar{\theta}_{1} s_{1, i} S_{3}(y) \pi_{0,0} \\
& +\left(p+\frac{\bar{p}}{z} a_{0}\right) \bar{\theta}_{1} s_{1, i} S_{3}(y) \bar{r} \phi_{1,1}(z) \\
& +\left(p+\frac{\bar{p}}{z} a_{0}\right) \bar{\theta}_{1} s_{1, i} S_{3}(y) \phi_{2,1}(z) \\
& +(\bar{p}+p \bar{\alpha}+z p \alpha) \bar{\theta}_{1} S_{3}(y) \phi_{1, i+1}(z) \\
& +(\bar{p}+p \bar{\alpha}+z p \alpha) \bar{\theta}_{1} S_{3}(y) \phi_{3, i, 1}(z) \\
& +(\bar{p}+p \bar{\alpha}+z p \alpha)\left[\frac{\phi_{3, i}(y, z)}{y}-\phi_{3, i, 1}(z)\right], \quad i \geq 1, \quad j \geq 1 .
\end{aligned}
$$

Next, multiplying (17), (18), (14), (20), and (21) by $x^{i}$ and summing over $i$, we get

$$
\begin{aligned}
\frac{x-\bar{p}}{x} \phi_{0}(x, z)= & \bar{p}\left[A(x)-a_{0}\right]\left(\bar{r} \phi_{1,1}(z)+\phi_{2,1}(z)\right) \\
& -p\left[A(x)-a_{0}\right] \pi_{0,0}-\bar{p} \phi_{0,1}(z)
\end{aligned}
$$

$$
\begin{aligned}
\frac{x-\theta_{1} \omega(z)}{x} \phi_{1}(x, z)= & \frac{\bar{p}}{z} \theta_{1} S_{1}(x) \phi_{0,1}(z) \\
& +p \theta_{1} S_{1}(x) \phi_{0}(1, z) \\
& +\left[\left(p+\frac{\bar{p}}{z} a_{0}\right) \theta_{1} S_{1}(x) \bar{r}-\theta \omega(z)\right] \\
& \times \phi_{1,1}(z)+\theta_{1} \omega(z) \psi_{1}(x, z) \\
& +\left(p+\frac{\bar{p}}{z} a_{0}\right) \theta_{1} S_{1}(x) \phi_{2,1}(z) \\
& +\left(1-\frac{a_{0}}{z}\right) p \theta_{1} S_{1}(x) \pi_{0,0},
\end{aligned}
$$

$$
\begin{aligned}
& \frac{x-\theta_{2} \omega(z)}{x} \phi_{2}(x, z)=r \omega(z) \theta_{2} S_{2}(x) \phi_{1,1}(z) \\
& -\theta_{2} \omega(z) \phi_{2,1}(z) \\
& +\theta_{2} \omega(z) \psi_{2}(x, z) \text {, } \\
& \frac{y-\omega(z)}{y} \phi_{3}(x, y, z) \\
& =p \bar{\theta}_{1} S_{1}(x) S_{3}(y) \phi_{0}(1, z) \\
& +\frac{\bar{p}}{z} \bar{\theta}_{1} S_{1}(x) S_{3}(y) \phi_{0,1}(z) \\
& +\left(p+\frac{\bar{p}}{z} a_{0}\right) \bar{\theta}_{1} S_{1}(x) S_{3}(y) \bar{r} \phi_{1,1}(z) \\
& +\left(p+\frac{\bar{p}}{z} a_{0}\right) \bar{\theta}_{1} S_{1}(x) S_{3}(y) \phi_{2,1}(z) \\
& +\omega(z)\left(\bar{\theta}_{1} S_{3}(y)-1\right) \psi_{1}(x, z) \\
& +\omega(z) \bar{\theta}_{1} S_{3}(y)\left(\frac{\phi_{1}(x, z)}{x}-\phi_{1,1}(z)\right) \\
& +\left(1-\frac{a_{0}}{z}\right) p \bar{\theta}_{1} S_{1}(x) S_{3}(y) \pi_{0,0}, \\
& \frac{y-\omega(z)}{y} \phi_{4}(x, y, z) \\
& =r \omega(z) \bar{\theta}_{2} S_{2}(x) S_{4}(y) \phi_{1,1}(z) \\
& +\omega(z)\left(\bar{\theta}_{2} S_{4}(y)-1\right) \psi_{2}(x, z) \\
& +\omega(z) \bar{\theta}_{2} S_{4}(y) \\
& \times\left(\frac{\phi_{2}(x, z)}{x}-\phi_{2,1}(z)\right),
\end{aligned}
$$

where $\omega(z)=\bar{p}+p \bar{\alpha}+z p \alpha$.

Choosing $x=1$ in (22), we can get $\phi_{0}(1, z)$ and putting $\phi_{0}(1, z)$ into (23), (25), we obtain

$$
\begin{aligned}
\frac{x-\theta_{1} \omega(z)}{x} \phi_{1}(x, z)= & \frac{\bar{p}(1-z) \theta_{1} S_{1}(x)}{z} \phi_{0,1}(z) \\
& -\frac{p a_{0}(1-z) \theta_{1} S_{1}(x)}{z} \pi_{0,0} \\
& +\left[\frac{z+\bar{p} a_{0}(1-z)}{z} \bar{r} S_{1}(x)-\omega(z)\right] \\
& \times \theta_{1} \phi_{1,1}(z)+\frac{z+\bar{p} a_{0}(1-z)}{z} \\
& \times S_{1}(x) \theta_{1} \phi_{2,1}(z)+\omega(z) \theta_{1} \psi_{1}(x, z),
\end{aligned}
$$




$$
\begin{aligned}
\frac{y-\omega(z)}{y} \phi_{3}(x, y, z)= & \frac{(1-z) \bar{p} \bar{\theta}_{1} S_{1}(x) S_{3}(y)}{z} \phi_{0,1}(z) \\
& -\frac{p a_{0}(1-z) \bar{\theta}_{1} S_{1}(x) S_{3}(y)}{z} \pi_{0,0} \\
& +\left[\frac{z+\bar{p} a_{0}(1-z)}{z} \bar{r} S_{1}(x)-\omega(z)\right] \\
& \times \bar{\theta}_{1} S_{3}(y) \phi_{1,1}(z) \\
& +\frac{z+\bar{p} a_{0}(1-z)}{z} \\
& \times \bar{r} S_{1}(x) \bar{\theta}_{1} S_{1}(x) S_{3}(y) \phi_{2,1}(z) \\
& +\frac{\omega(z) \bar{\theta}_{1} S_{3}(y)}{x} \phi_{1}(x, z)-\omega(z) \\
& \times\left(1-\bar{\theta}_{1} S_{3}(y)\right) \psi_{1}(x, z) .
\end{aligned}
$$

Letting $y=\omega(z)$ in (26), (28), we obtain

$$
\begin{aligned}
\psi_{2}(x, z)= & \frac{S_{4}(\omega(z)) \bar{\theta}_{2}}{\omega(z)\left[1-\bar{\theta}_{2} S_{4}(\omega(z))\right]} \\
& \times\left\{r \omega(z) S_{2}(x) \phi_{1,1}(z)\right. \\
& \left.-\omega(z) \phi_{2,1}(z)+\frac{\omega(z)}{x} \phi_{2}(x, z)\right\}, \\
\psi_{1}(x, z)= & \frac{S_{3}(\omega(z)) \bar{\theta}_{1}}{\omega(z)\left[1-\bar{\theta}_{1} S_{3}(\omega(z))\right]} \\
& \times\left\{\frac{(1-z) \bar{p} S_{1}(x)}{z} \phi_{0,1}(z)\right. \\
& \quad-\frac{p a_{0}(1-z) S_{1}(x)}{z} \pi_{0,0} \\
& +\left[\frac{z+\bar{p} a_{0}(1-z)}{z} \bar{r} S_{1}(x)-\omega(z)\right] \phi_{1,1}(z) \\
& +\frac{z+\bar{p} a_{0}(1-z)}{z} \\
& \left.\times S_{1}(x) \phi_{2,1}(z)+\frac{\omega(z)}{x} \phi_{1}(x, z)\right\} .
\end{aligned}
$$

Substituting (29), (30) into (24), (27), we obtain

$$
\begin{aligned}
& \frac{x-\varphi_{2}[w(z)]}{x}\left[1-\bar{\theta}_{2} S_{4}(w(z))\right] \phi_{2}(x, z) \\
& =\theta_{2}\left\{r \omega(z) S_{2}(x) \phi_{1,1}(z)-\omega(z) \phi_{2,1}(z)\right\},
\end{aligned}
$$

$$
\begin{gathered}
\frac{x-\varphi_{1}[w(z)]}{x}\left[1-\bar{\theta}_{1} S_{3}(w(z))\right] \phi_{1}(x, z) \\
=\theta_{1}\left\{\frac{1-z}{z} \bar{p} S_{1}(x) \phi_{0,1}(z)-\frac{1-z}{z} p a_{0} S_{1}(x) \pi_{0,0}\right. \\
+\left[\frac{z+\bar{p} a_{0}(1-z)}{z} S_{1}(x) \bar{r}-\omega(z)\right] \phi_{1,1}(z) \\
\left.+\frac{z+\bar{p} a_{0}(1-z)}{z} S_{1}(x) \phi_{2,1}(z)\right\},
\end{gathered}
$$

where functions

$$
\varphi_{1}(z)=\frac{\theta_{1} z}{1-\bar{\theta}_{1} S_{3}(z)}, \quad \varphi_{2}(z)=\frac{\theta_{2} z}{1-\bar{\theta}_{2} S_{4}(z)} .
$$

Letting $x=\bar{p}$ in (22), $x=\varphi_{2}(\omega(z))$ in (31), and $x=\varphi_{1}(\omega(z))$ in (32), we get

$$
\begin{aligned}
& p\left[A(\bar{p})-a_{0}\right] \pi_{0,0}= \bar{p}\left[A(\bar{p})-a_{0}\right] \\
& \times\left[\bar{r} \phi_{1,1}(z)+\phi_{2,1}(z)\right] \\
&-\bar{p} \phi_{0,1}(z), \\
& \phi_{2,1}(z)=r S_{2}\left[\varphi_{2}(\omega(z))\right] \phi_{1,1}(z), \\
& \frac{p a_{0}(1-z) S_{1}\left[\varphi_{1}(\omega(z))\right]}{z} \pi_{0,0} \\
&=\frac{\bar{p}(1-z) S_{1}\left[\varphi_{1}(\omega(z))\right]}{z} \phi_{0,1}(z) \\
&+\left[\frac{z+\bar{p} a_{0}(1-z) \bar{r} S_{1}\left[\varphi_{1}(\omega(z))\right]}{z}\right. \\
&+\frac{z+\bar{p} a_{0}(1-z) S_{1}\left[\varphi_{1}(\omega(z))\right]}{z} \phi_{2,1}(z) .
\end{aligned}
$$

Solving (34) we obtain the auxiliary generating functions $\phi_{0,1}(z), \phi_{1,1}(z)$, and $\phi_{2,1}(z)$ as follows:

$$
\begin{aligned}
\phi_{0,1}(z)=( & p z\left(A(\bar{p})-a_{0}\right) \\
& \left.\times\left[\omega(z)-S_{1}\left[\varphi_{1}(\omega(z))\right]\left[\bar{r}+r S_{2}\left[\varphi_{2}(\omega(z))\right]\right]\right]\right) \\
& \times(\Upsilon(z))^{-1} \times \frac{\pi_{0,0}}{\bar{p}}
\end{aligned}
$$




$$
\begin{aligned}
\phi_{1,1}(z)= & \left(p A(\bar{p})(1-z) S_{1}\right. \\
& \left.\times\left[\varphi_{1}(\omega(z))\right]\left[\bar{r}+r S_{2}\left[\varphi_{2}(\omega(z))\right]\right]\right) \\
& \times(\Upsilon(z))^{-1} \times \pi_{0,0}, \\
\phi_{2,1}(z)= & r S_{2}\left[\varphi_{2}(\omega(z))\right] \\
& \times\left(p A(\bar{p})(1-z) S_{1}\right. \\
& \left.\times\left[\varphi_{1}(\omega(z))\right]\left[\bar{r}+r S_{2}\left[\varphi_{2}(\omega(z))\right]\right]\right) \\
& \times(\Upsilon(z))^{-1} \times \pi_{0,0},
\end{aligned}
$$

where

$$
\begin{aligned}
\Upsilon(z)= & {[z+\bar{p} A(\bar{p})(1-z)] } \\
& \times S_{1}\left[\varphi_{1}(\omega(z))\right]\left[\bar{r}+r S_{2}\left[\varphi_{2}(\omega(z))\right]\right]-z \omega(z) .
\end{aligned}
$$

Next, we give two lemmas which will be used later on and their proof can be readily obtained. Thus, they are omitted.

Lemma 1. The inequality

$$
\begin{aligned}
\bar{p} A(\bar{p}) & (1-z) S_{1}\left[\varphi_{1}(\omega(z))\right]\left[\bar{r}+r S_{2}\left[\varphi_{2}(\omega(z))\right]\right] \\
& -z\left[\omega(z)-S_{1}\left[\varphi_{1}(\omega(z))\right]\left[\bar{r}+r S_{2}\left[\varphi_{2}(\omega(z))\right]\right]\right]>0
\end{aligned}
$$

holds, for $0 \leq z<1$, if

$$
\rho_{1}+r \rho_{2}<p+\frac{\bar{p} A(\bar{p})}{\alpha}
$$

where $\rho_{1}=p \beta_{1,1}\left(1+\left(\bar{\theta}_{1} / \theta_{1}\right) \beta_{3,1}\right), \rho_{2}=p \beta_{2,1}\left(1+\left(\bar{\theta}_{2} / \theta_{2}\right) \beta_{4,1}\right)$.

Lemma 2. The following limits are positive if $\rho_{1}+r \rho_{2}<p+$ $\bar{p} A(\bar{p}) / \alpha$ as follows:

$$
\begin{gathered}
\lim _{z \rightarrow 1} \frac{1-z}{\Upsilon(z)}=\frac{1}{\bar{p} A(\bar{p})+\alpha p-\alpha\left(\rho_{1}+r \rho_{2}\right)}, \\
\lim _{z \rightarrow 1} \frac{\omega(z)-S_{1}\left[\varphi_{1}(\omega(z))\right]\left[\bar{r}+r S_{2}\left[\varphi_{2}(\omega(z))\right]\right]}{\Upsilon(z)} \\
=\frac{\alpha\left(\rho_{1}+r \rho_{2}-p\right)}{\bar{p} A(\bar{p})+\alpha p-\alpha\left(\rho_{1}+r \rho_{2}\right)} .
\end{gathered}
$$

From above Lemmas, we can easily get that $\phi_{0,1}(z)$ and $\phi_{1,1}(z)$ are defined for $z \in[0,1)$ and can be extended by continuity, for $z=1$, if $\rho_{1}+r \rho_{2}<p+\bar{p} A(\bar{p}) / \alpha$. Then, we can obtain the generating functions of the stationary distribution of the system which are given by the following theorem.
Theorem 3. If $\rho_{1}+r \rho_{2}<p+\bar{p} A(\bar{p}) / \alpha$, the stationary distribution of the Markov chain $\left\{Y_{m}, m=1,2, \ldots\right\}$ has the following generating functions:

$$
\begin{aligned}
\phi_{0}(x, z)= & p x z\left\{\omega(z)-S_{1}\left[\varphi_{1}(\omega(z))\right]\left[\bar{r}+r S_{2}\left[\varphi_{2}(\omega(z))\right]\right]\right\} \\
& \times(\Upsilon(z))^{-1} \\
& \times \frac{A(x)-A(\bar{p})}{x-\bar{p}} \pi_{0,0},
\end{aligned}
$$$$
\phi_{1}(x, z)=\frac{S_{1}(x)-S_{1}\left[\varphi_{1}(\omega(z))\right]}{x-\varphi_{1}(\omega(z))}
$$

$$
\times \frac{p \omega(z) A(\bar{p})(1-z) x \theta_{1}}{\Upsilon(z)\left[1-\bar{\theta}_{1} S_{3}(\omega(z))\right]} \pi_{0,0},
$$$$
\phi_{2}(x, z)=\frac{S_{2}(x)-S_{2}\left[\varphi_{2}(\omega(z))\right]}{x-\varphi_{2}(\omega(z))}
$$

$$
\times \frac{r p \omega(z) A(\bar{p})(1-z) x \theta_{2} S_{1}\left[\varphi_{1}(\omega(z))\right]}{\Upsilon(z)\left[1-\bar{\theta}_{2} S_{4}(\omega(z))\right]} \pi_{0,0},
$$

$$
\begin{aligned}
\phi_{3}(x, y, z)= & \frac{S_{1}(x)-S_{1}\left[\varphi_{1}(\omega(z))\right]}{x-\varphi_{1}(\omega(z))} \\
& \times \frac{S_{3}(y)-S_{3}(\omega(z))}{y-\omega(z)} \\
& \times \frac{p \omega(z) A(\bar{p})(1-z) x y \bar{\theta}_{1}}{\Upsilon(z)\left[1-\bar{\theta}_{1} S_{3}(\omega(z))\right]} \pi_{0,0}, \\
\phi_{4}(x, y, z)= & \frac{S_{2}(x)-S_{2}\left[\varphi_{2}(\omega(z))\right]}{x-\varphi_{2}(\omega(z))} \\
& \times \frac{S_{4}(y)-S_{4}(\omega(z))}{y-\omega(z)} \\
& \times \frac{r p \omega(z) A(\bar{p})(1-z) x y \bar{\theta}_{2} S_{1}\left[\varphi_{1}(\omega(z))\right]}{\Upsilon(z)\left[1-\bar{\theta}_{2} S_{4}(\omega(z))\right]} \\
& \times \pi_{0,0}, \quad
\end{aligned}
$$

where

$$
\pi_{0,0}=\frac{\bar{p} A(\bar{p})+\alpha p-\alpha\left(\rho_{1}+r \rho_{2}\right)}{A(\bar{p})\left(\bar{p}+\alpha p+\bar{\alpha}\left(\rho_{1}+r \rho_{2}\right)\right)} .
$$

Proof. Substituting (35)-(37) into (22), (31) and (32), yields $\phi_{0}(x, z), \phi_{2}(x, z)$, and $\phi_{1}(x, z)$ in Theorem 3. Further, substituting (36), (37), (43), and (31) into (29) and (30), respectively, we get

$$
\begin{aligned}
\psi_{2}(x, z)= & \frac{S_{4}(\omega(z))}{\omega(z)} \frac{S_{2}(x)-S_{2}\left[\varphi_{2}(\omega(z))\right]}{x-\varphi_{2}(\omega(z))} \\
& \times \frac{r p \omega(z) A(\bar{p})(1-z) x \bar{\theta}_{2} S_{1}\left[\varphi_{1}(\omega(z))\right]}{\Upsilon(z)\left[1-\bar{\theta}_{2} S_{4}(\omega(z))\right]} \pi_{0,0},
\end{aligned}
$$




$$
\begin{aligned}
\psi_{1}(x, z)= & \frac{S_{3}(\omega(z))}{\omega(z)} \frac{S_{1}(x)-S_{1}\left[\varphi_{1}(\omega(z))\right]}{x-\varphi_{1}(\omega(z))} \\
& \times \frac{p \omega(z) A(\bar{p})(1-z) x \bar{\theta}_{1}}{\Upsilon(z)\left[1-\bar{\theta}_{1} S_{3}(\omega(z))\right]} \pi_{0,0} .
\end{aligned}
$$

Inserting (35)-(37), (48)-(49) into (26) and (28), respectively, we can get $\phi_{4}(x, y, z)$ and $\phi_{3}(x, y, z)$ in Theorem 3 . From normalizing condition (9), which can be written as

$$
\pi_{0,0}+\phi_{0}(1,1)+\sum_{i=1}^{2} \phi_{i}(1,1)+\sum_{j=3}^{4} \phi_{j}(1,1,1)=1 \text {, }
$$

we can find the unknown constant $\pi_{0,0}$ in Theorem 3 .

Based on Theorem 3, we can easily obtain the marginal generating functions of the number of customers when the server is in various states and some performance measures. They are summarized in the following Corollary. Their proofs are very easy, and thus are omitted.

Corollary 4. (1) The marginal generating function of the number of customers in the orbit when the server is idle is given by

$$
\begin{aligned}
& \pi_{0,0}+\phi_{0}(1, z) \\
&=A(\bar{p})\left\{\frac{S_{1}\left[\varphi_{1}(\omega(z))\right]\left[\bar{r}+r S_{2}\left[\varphi_{2}(\omega(z))\right]\right](1-z) \bar{p}}{\Upsilon(z)}\right. \\
&-\left(z\left(\omega(z)-S_{1}\left[\varphi_{1}(\omega(z))\right]\left[\bar{r}+r S_{2}\left[\varphi_{2}(\omega(z))\right]\right]\right)\right) \\
&\left.\quad \times(\Upsilon(z))^{-1}\right\} \times \pi_{0,0} .
\end{aligned}
$$

(2) The marginal generating function of the number of customers in the orbit when the server is busy with FPS is given by

$$
\begin{aligned}
\phi_{1}(1, z)= & \frac{1-S_{1}\left[\varphi_{1}(\omega(z))\right]}{1-\varphi_{1}(\omega(z))} \\
& \times \frac{(1-z) p A(\bar{p}) \omega(z) \theta_{1}}{\Upsilon(z)\left(1-\bar{\theta}_{1} S_{3}(\omega(z))\right)} \pi_{0,0} .
\end{aligned}
$$

(3) The marginal generating function of the number of customers in the orbit when the server is busy with SPS is given by

$$
\begin{aligned}
\phi_{2}(1, z)= & \frac{1-S_{2}\left[\varphi_{2}(\omega(z))\right]}{1-\varphi_{2}(\omega(z))} \\
& \times \frac{r p \omega(z) A(\bar{p})(1-z) \theta_{2} S_{1}\left[\varphi_{1}(\omega(z))\right]}{\Upsilon(z)\left[1-\bar{\theta}_{2} S_{4}(\omega(z))\right]} \pi_{0,0} .
\end{aligned}
$$

(4) The marginal generating function of the number of customers in the orbit when the server is under repair during FPS is given by

$$
\begin{aligned}
\phi_{3}(1,1, z)= & \frac{1-S_{1}\left[\varphi_{1}(\omega(z))\right]}{1-\varphi_{1}(\omega(z))} \\
& \times \frac{1-S_{3}(\omega(z))}{\alpha \Upsilon(z)} \frac{A(\bar{p}) \omega(z) \bar{\theta}_{1}}{1-\bar{\theta}_{1} S_{3}(\omega(z))} \pi_{0,0} .
\end{aligned}
$$

(5) The marginal generating function of the number of customers in the orbit when the server is under repair during SPS is given by

$$
\begin{aligned}
\phi_{4}(1,1, z)= & \frac{1-S_{2}\left[\varphi_{2}(\omega(z))\right]}{1-\varphi_{2}(\omega(z))} \frac{1-S_{4}(\omega(z))}{\alpha \Upsilon(z)} \\
& \times \frac{A(\bar{p}) \omega(z) \bar{\theta}_{2} r S_{1}\left[\varphi_{1}(\omega(z))\right]}{1-\bar{\theta}_{2} S_{4}(\omega(z))} \pi_{0,0} .
\end{aligned}
$$

(6) The probability generating function of the orbit size (i.e., of the variable $N$ ) is given by

$$
\begin{aligned}
& \Psi(z)= \pi_{0,0}+\phi_{0}(1, z)+\sum_{i=1}^{2} \phi_{i}(1, z)+\sum_{j=3}^{4} \phi_{j}(1,1, z) \\
&=\left(A ( \overline { p } ) \left\{\omega(z)(1-\alpha z)-\bar{\alpha} S_{1}\right.\right. \\
&\left.\left.\quad \times\left[\varphi_{1}(\omega(z))\right]\left[\bar{r}+r S_{2}\left[\varphi_{2}(\omega(z))\right]\right]\right\}\right) \\
& \quad \times(\alpha \Upsilon(z))^{-1} \times \pi_{0,0} .
\end{aligned}
$$

(7) The probability generating function of the number of customers in the system (i.e., of the variable L) is given by

$$
\begin{aligned}
\Phi(z)= & \pi_{0,0}+\phi_{0}(1, z)+z \sum_{i=1}^{2} \phi_{i}(1, z)+z \sum_{j=3}^{4} \phi_{j}(1,1, z) \\
= & \left(A ( \overline { p } ) \left\{\bar{\alpha} z \omega(z)+S_{1}\left[\varphi_{1}(\omega(z))\right]\right.\right. \\
& \left.\left.\quad \times\left[\bar{r}+r S_{2}\left[\varphi_{2}(\omega(z))\right]\right][\omega(z)(1-z)-\bar{\alpha}]\right\}\right) \\
& \times(\alpha \Upsilon(z))^{-1} \times \pi_{0,0} .
\end{aligned}
$$

Corollary 5. (1) The system is idle with probability

$$
\pi_{0,0}=\frac{\bar{p} A(\bar{p})+\alpha p-\alpha\left(\rho_{1}+r \rho_{2}\right)}{A(\bar{p})\left(\bar{p}+\alpha p+\bar{\alpha}\left(\rho_{1}+r \rho_{2}\right)\right)} .
$$

(2) The system is occupied with probability

$$
\begin{aligned}
\phi_{0}(1,1)+\sum_{i=1}^{2} \phi_{i}(1,1)+\sum_{j=3}^{4} \phi_{j}(1,1,1) \\
=\frac{A(\bar{p})\left(\alpha p+\bar{\alpha}\left(\rho_{1}+r \rho_{2}\right)\right)-\alpha\left(p-\rho_{1}-r \rho_{2}\right)}{A(\bar{p})\left(\bar{p}+\alpha p+\bar{\alpha}\left(\rho_{1}+r \rho_{2}\right)\right)} .
\end{aligned}
$$


(3) The probability that the server is idle is

$$
\pi_{0,0}+\phi_{0}(1,1)=\frac{\bar{p}+\alpha p-\alpha\left(\rho_{1}+r \rho_{2}\right)}{\bar{p}+\alpha p+\bar{\alpha}\left(\rho_{1}+r \rho_{2}\right)} .
$$

(4) The probability that the server is busy with FPS is

$$
\phi_{1}(1,1)=\frac{p \beta_{1,1}}{\bar{p}+\alpha p+\bar{\alpha}\left(\rho_{1}+r \rho_{2}\right)} .
$$

(5) The probability that the server is busy with SPS is

$$
\phi_{2}(1,1)=\frac{r p \beta_{2,1}}{\bar{p}+\alpha p+\bar{\alpha}\left(\rho_{1}+r \rho_{2}\right)} .
$$

(6) The probability that the server is under repair during FPS is

$$
\phi_{3}(1,1,1)=\frac{p \beta_{1,1} \beta_{3,1}\left(\bar{\theta}_{1} / \theta_{1}\right)}{\bar{p}+\alpha p+\bar{\alpha}\left(\rho_{1}+r \rho_{2}\right)} .
$$

(7) The probability that the server is under repair during SPS is

$$
\phi_{4}(1,1,1)=\frac{p \beta_{2,1} \beta_{4,1}\left(\bar{\theta}_{2} / \theta_{2}\right)}{\bar{p}+\alpha p+\bar{\alpha}\left(\rho_{1}+r \rho_{2}\right)} .
$$

(8) The mean number of customers in the orbit is

$$
\begin{aligned}
E(N)= & \left(2 \alpha [ p - \rho _ { 1 } - r \rho _ { 2 } ] \left[\bar{p} A(\bar{p})\left(1+\bar{\alpha}\left(\rho_{1}+r \rho_{2}\right)\right)\right.\right. \\
& \left.\left.-\left(\bar{p}+\bar{\alpha}\left(\rho_{1}+r \rho_{2}\right)\right)\right]\right) \\
\times & \left(2\left[\bar{p}+\alpha p+\bar{\alpha}\left(\rho_{1}+r \rho_{2}\right)\right]\right. \\
& \left.\times\left[\bar{p} A(\bar{p})+\alpha\left(p-\rho_{1}-r \rho_{2}\right)\right]\right)^{-1} \\
+ & \left(\alpha p^{2} \tau[\alpha+\bar{\alpha} \bar{p} A(\bar{p})]\right) \\
\times & \left(2\left[\bar{p}+\alpha p+\bar{\alpha}\left(\rho_{1}+r \rho_{2}\right)\right]\right. \\
& \left.\times\left[\bar{p} A(\bar{p})+\alpha\left(p-\rho_{1}-r \rho_{2}\right)\right]\right)^{-1}
\end{aligned}
$$

where

$$
\begin{aligned}
\tau= & \beta_{1,2}\left(1+\frac{\bar{\theta}_{1}}{\theta_{1}} \beta_{3,1}\right)^{2} \\
& +\beta_{1,1}\left[\frac{\bar{\theta}_{1}}{\theta_{1}} \beta_{3,2}+2 \frac{\bar{\theta}_{1}}{\theta_{1}} \beta_{3,1}\left(1+\frac{\bar{\theta}_{1}}{\theta_{1}} \beta_{3,1}\right)\right] \\
& +r \beta_{2,2}\left(1+\frac{\bar{\theta}_{2}}{\theta_{2}} \beta_{4,1}\right)^{2} \\
& +r \beta_{2,1}\left[\frac{\bar{\theta}_{2}}{\theta_{2}} \beta_{4,2}+2 \frac{\bar{\theta}_{2}}{\theta_{2}} \beta_{4,1}\left(1+\frac{\bar{\theta}_{1}}{\theta_{1}} \beta_{4,1}\right)\right] \\
& +2 r \beta_{1,1} \beta_{2,1}\left(1+\frac{\bar{\theta}_{1}}{\theta_{1}} \beta_{3,1}\right)\left(1+\frac{\bar{\theta}_{2}}{\theta_{2}} \beta_{4,1}\right) .
\end{aligned}
$$

(9) The arrival rate to the orbit is given by

$$
\begin{aligned}
P_{\text {Orbit }} & =\alpha p\left[\sum_{i=1}^{2} \phi_{i}(1,1)+\sum_{j=3}^{4} \phi_{j}(1,1,1)\right] \\
& =\frac{\alpha p\left(\rho_{1}+r \rho_{2}\right)}{\bar{p}+\alpha p+\bar{\alpha}\left(\rho_{1}+r \rho_{2}\right)} .
\end{aligned}
$$

(10) The arrival rate to the system is given by

$$
\begin{aligned}
P_{\text {System }}= & p\left[\pi_{0,0}+\phi_{0}(1,1)\right] \\
& +\alpha p\left[\sum_{i=1}^{2} \phi_{i}(1,1)+\sum_{j=1}^{2} \phi_{j}(1,1,1)\right] \\
= & \frac{p(\bar{p}+\alpha p)}{\bar{p}+\alpha p+\bar{\alpha}\left(\rho_{1}+r \rho_{2}\right)} .
\end{aligned}
$$

(11) The loss probability of a customer is

$$
\begin{aligned}
P_{\text {Loss }} & =p \bar{\alpha}\left[\sum_{i=1}^{2} \phi_{i}(1,1)+\sum_{j=3}^{4} \phi_{j}(1,1,1)\right] \\
& =\frac{p \bar{\alpha}\left(\rho_{1}+r \rho_{2}\right)}{\bar{p}+\alpha p+\bar{\alpha}\left(\rho_{1}+r \rho_{2}\right)} .
\end{aligned}
$$

Remark 6 (special cases). In the remark, we consider some special cases of our model.

(i) When $\theta_{1}=1, r=0$, the present model reduced to a discrete-time $G e o / G / 1$ retrial queue with balking customer. In this case, the generating function in Theorem 3 reduces to

$\phi_{0}(x, z)$

$$
\begin{aligned}
= & \frac{p x z\left(\omega(z)-S_{1}(\omega(z))\right)}{\bar{p} A(\bar{p})(1-z) S_{1}(\omega(z))-z\left[\omega(z)-S_{1}(\omega(z))\right]} \\
& \times \frac{A(x)-A(\bar{p})}{x-\bar{p}} \pi_{0,0},
\end{aligned}
$$

$\phi_{1}(x, z)$

$$
\begin{aligned}
= & \frac{S_{1}(x)-S_{1}\left[\varphi_{1}(\omega(z))\right]}{x-\omega(z)} \\
& \times \frac{p \omega(z) A(\bar{p})(1-z) x}{\bar{p} A(\bar{p})(1-z) S_{1}(\omega(z))-z\left[\omega(z)-S_{1}(\omega(z))\right]} \pi_{0,0},
\end{aligned}
$$

where

$$
\pi_{0,0}=\frac{\bar{p} A(\bar{p})+\alpha p-\alpha \rho}{A(\bar{p})(\bar{p}+\alpha p+\bar{\alpha} \rho)}, \quad \rho=p \beta_{1,1},
$$

which coincides with the generating functions of Theorem 3.1 in Aboul-Hassan et al. [11]. 
(ii) When $\theta_{1}=1, r=0$, and $\alpha=1$, the present model reduced to a discrete-time $G e o / G / 1$ retrial queue with general retrial times. In this case, the generating function in Theorem 3 reduces to

$$
\begin{aligned}
& \phi_{0}(x, z) \\
& =\frac{p x z\left(\gamma(z)-S_{1}(\gamma(z))\right)}{\bar{p} A(\bar{p})(1-z) S_{1}(\gamma(z))-z\left[\gamma(z)-S_{1}(\gamma(z))\right]} \quad(72) \\
& \quad \times \frac{A(x)-A(\bar{p})}{x-\bar{p}} \pi_{0,0}, \\
& \phi_{1}(x, z) \\
& =\frac{S_{1}(x)-S_{1}[\gamma(z)]}{x-\gamma(z)} \\
& \quad \times \frac{p \gamma(z) A(\bar{p})(1-z) x}{\bar{p} A(\bar{p})(1-z) S_{1}(\gamma(z))-z\left[\gamma(z)-S_{1}(\gamma(z))\right]} \pi_{0,0},
\end{aligned}
$$

where

$$
\pi_{0,0}=\frac{\bar{p} A(\bar{p})+p-\rho}{A(\bar{p})}, \quad \rho=p \beta_{1,1},
$$

which coincides with the generating functions of Theorem 1 in Atencia and Moreno [7].

\section{Stochastic Decomposition}

The property of stochastic decomposition was studied firstly in queueing system with vacations; see Fuhrmann and Cooper [25]. Artalejo and Falin [26] generalized the concept of stochastic decomposition to retrial queueing system. In this section, we give a stochastic decomposition result of the system size distribution in our model. As a consequence of the stochastic decomposition result, we provide upper and lower estimates for the distance between the steady-state distribution of our discrete-time queueing system and the corresponding system without retrials.

Theorem 7. The total number of customers in the system $(L)$ can be represented as the sum of two independent random variables, one of which is the number of customers in the unreliable Geo/G/1/ $\infty$ queue with customer balking and second optional service $\left(L_{0}\right)$ and the other is the number of repeated customers given that the server is idle or down $\left(M_{0}\right)$. That is, $L=L_{0}+M_{0}$.

Proof. It is easy to show that $\Phi(z)$ can be represented by $\Phi(z)=\Phi_{1}(z) \Phi_{2}(z)$, where

$$
\begin{aligned}
& \Phi_{1}(z) \\
& =\left(\bar{\alpha} z \omega(z)+S_{1}\left[\varphi_{1}(\omega(z))\right]\left[\overline{r+r} S_{2}\left[\varphi_{2}(\omega(z))\right]\right]\right. \\
& \quad \times[\omega(z)(1-z)-\bar{\alpha}]) \times(\alpha \Omega(z))^{-1} \\
& \quad \times \frac{\bar{p}+p \alpha-\alpha\left(\rho_{1}+r \rho_{2}\right)}{\bar{p}+p \alpha+\bar{\alpha}\left(\rho_{1}+r \rho_{2}\right)}
\end{aligned}
$$

is the generating function of $G e o / G / 1$ queue with balking customer second optional service and unreliable server in special case (i) and $\Phi_{2}(z)$ given by

$$
\begin{aligned}
\Phi_{2}(z)= & \sum_{k=0}^{\infty} P\left(M_{0}=k\right) z^{k} \\
= & \left((\bar{p}+p z) S_{1}\left[\varphi_{1}(\omega(z))\right]\left[\bar{r}+r S_{2}\left[\varphi_{2}(\omega(z))\right]\right]\right. \\
& \quad-z \omega(z)) \times(\Upsilon(z))^{-1} \\
& \times \frac{\bar{p} A(\bar{p})+\alpha p-\alpha\left(\rho_{1}+r \rho_{2}\right)}{\bar{p}+\alpha p-\alpha\left(\rho_{1}+r \rho_{2}\right)} \\
= & \frac{\pi_{0,0}+\phi_{0}(1, z)}{\pi_{0,0}+\phi_{0}(1,1)}
\end{aligned}
$$

is the probability generating function of the number of customers in the orbit given that the server is idle. This completes the proof.

The property of stochastic decomposition can be applied to give a measure of the proximity between the steady-state distributions of the unreliable $G e o / G / 1$ queue with balking and our queueing model. The result is summarized in the following theorem.

Theorem 8. The following inequalities hold:

$$
\begin{aligned}
& \frac{2 \alpha\left[p-\left(\rho_{1}+r \rho_{2}\right)\right][A(\bar{p})-1]}{A(\bar{p})\left[\bar{p}+\alpha p+\bar{\alpha}\left(\rho_{1}+r \rho_{2}\right)\right]} \\
& \leq \sum_{j=0}^{\infty}\left|P(L=j)-P\left(L_{0}=j\right)\right| \\
& \leq \frac{2 \alpha\left[p-\left(\rho_{1}+r \rho_{2}\right)\right][A(\bar{p})-1]}{A(\bar{p})\left[\bar{p}+\alpha p-\alpha\left(\rho_{1}+r \rho_{2}\right)\right]} .
\end{aligned}
$$

The proof which is similar to Aboul-Hassan et al. [11] is omitted.

\section{Numerical Examples}

In this section, we present some numerical examples to study the effect of the system parameters on the mean orbit size. We assume that $p$ is the probability that a customer arrives to the system and $q$ is probability that a customer receives the second phase service. Moreover, the retrial times are geometric distributions with parameters $r$ and thier generating function is $A(x)=(1-r) /(1-r x)$. It is also assumed that the service times during the FPS and SPS are geometric distributions with means $\beta_{1,1}$, and $\beta_{2,1}$, respectively, and the repair times for the FPS and SPS are geometric distributions with means $\beta_{3,1}$ and $\beta_{4,1}$, respectively.

In Figures 1 and 2, the mean number of customers in the orbit $E(N)$ is plotted against $q$. We present three curves which correspond to $\alpha=0.5,0.7,0.9$ and $\theta_{1}=0.5,0.6,0.7$, respectively, in Figures 1 and 2. We observe that $E(N)$ 


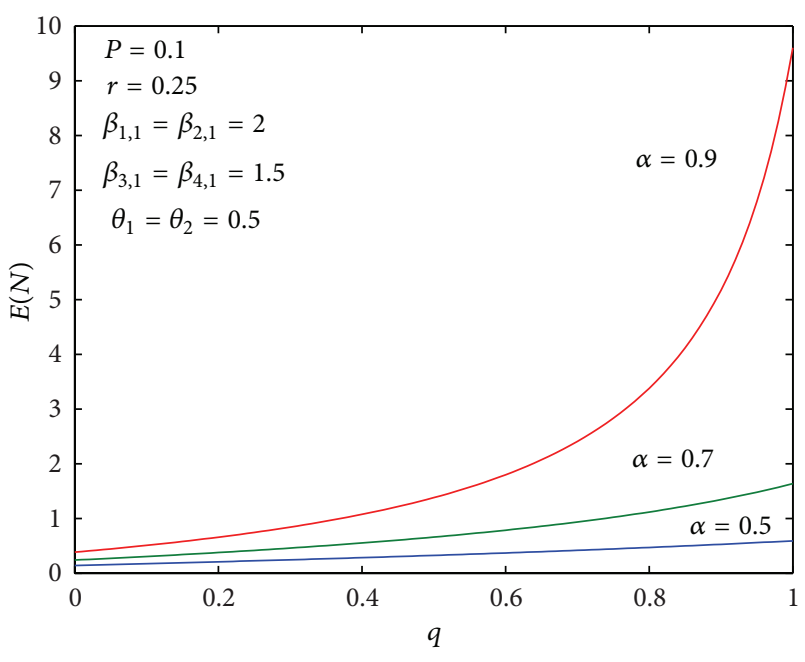

FIgURE 1: $E(N)$ versus $q$ for different $\alpha$.

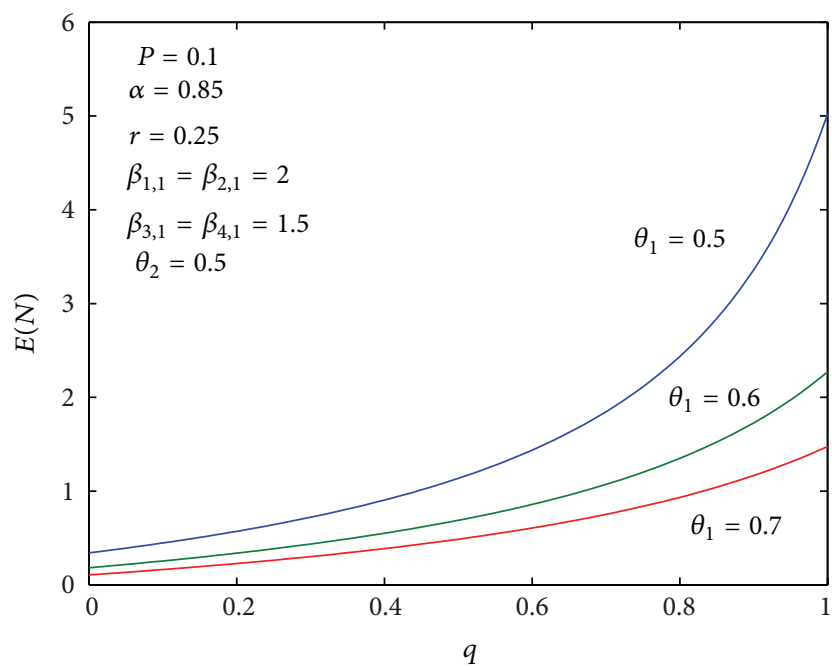

Figure 2: $E(N)$ versus $q$ for different $R$.

increases with the value of $q$. It is easily explained due to the fact that a customer choosing to receive the second-optional service increases the mean sojourn time. It is also shown that $E(N)$ is increasing as a function of $\alpha$ and decreases with the value $\theta_{1}$ which agree with the intuitive expectations.

In Figures 3 and 4, the mean number of customers in the orbit $E(N)$ is plotted against $r$ which is the probability that the retrial fails. We choose $p=0.1, \alpha=0.9, \beta_{1,1}=$ $\beta_{2,1}=2$, and $\theta_{1}=\theta_{2}=0.5$ in general. Figures 3 and 4 show that $E(N)$ exhibits a stable increasing when $r$ approximates a threshold value $\gamma$ (when $r \leq \gamma$ the system is stable) and then a sharp increasing with the increasing of $r$. It is also shown in Figures 3 and 4 that $E(N)$ is increasing with the mean repair time $\beta_{3,1}$ and $\beta_{4,1}$, respectively, which agree with the intuitive expectations.

From the curves in Figures 1 and 2, we can also obtain the comparative results between our model and the corresponding discrete-time queueing model without second optional service and server breakdowns. Figure 2 also shows that

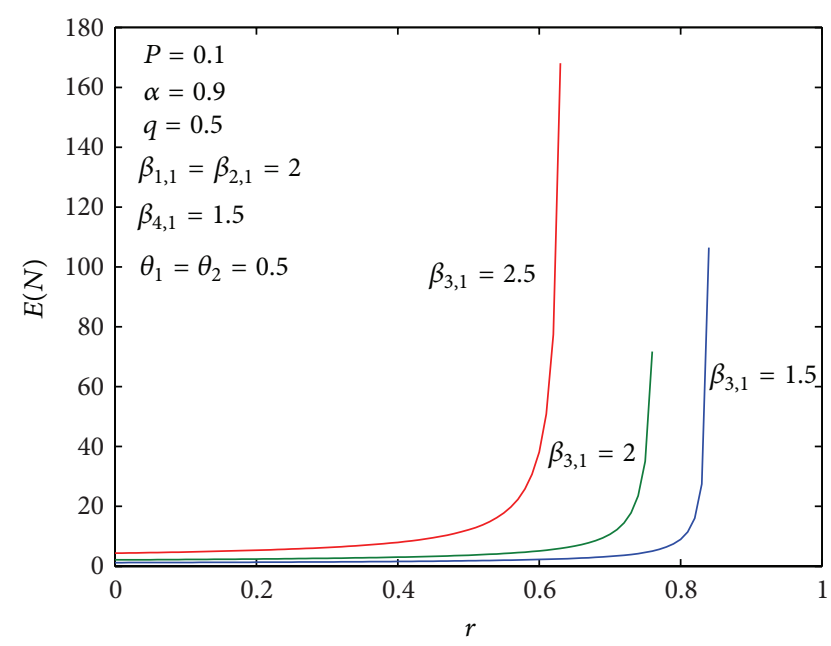

Figure 3: $E(N)$ versus $r$ for different $\theta_{1}$.

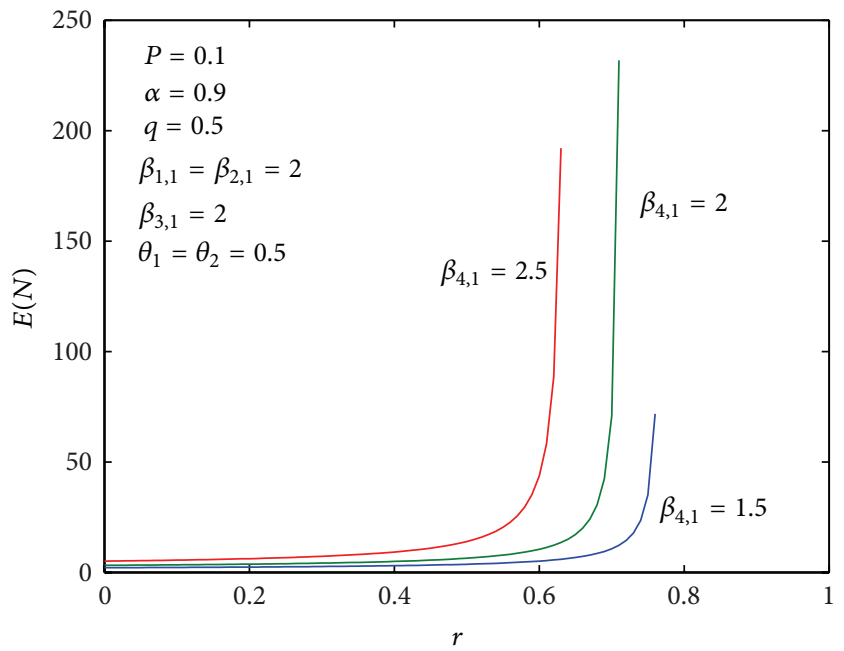

FIgURE 4: $E(N)$ versus $r$ for different $\beta_{3,1}$.

the mean number of customers in the orbit $E(N)$ increases significantly compared with the model of Aboul-Hassan et al. [11]. That is, our simulation results show that the breakdown of the server has a heavy impact on the performance measures of the discrete-time retrial queueing model with balking customer. Moreover, it is also shown in our simulation results that the impact of parameter $q$ on $E(N)$ is more noticeable as the probability that a customer may not balk increases. In addition, Figures 3 and 4 show how the mean repair time affects the mean customer of customers in the orbit. In general, compared with the previous model of Aboul-Hassan et al. [11] and Atencia and Moreno [24], our simulation results show the impact of various practical factors on the mean number of customers in the orbit $E(N)$ simultaneously.

\section{References}

[1] G. Falin, "A survey of retrial queues," Queueing Systems, vol. 7, no. 2, pp. 127-167, 1990. 
[2] G. I. Falin and J. G. C. Templeton, Retrial Queues, Chapman and Hall, New York, NY, USA, 1997.

[3] J. R. Artalejo and A. Gómez-Corral, Retrial Queueing Systems: A Computational Approach, Springer, Berlin, Germany, 2008.

[4] J. R. Artalejo, "Accessible bibliography on retrial queues: progress in 2000-2009," Mathematical and Computer Modelling, vol. 51, no. 9-10, pp. 1071-1081, 2010.

[5] A. Gómez-Corral, "A bibliographical guide to the analysis of retrial queues through matrix analytic techniques," Annals of Operations Research, vol. 141, pp. 163-191, 2006.

[6] T. Yang and H. Li, "On the steady-state queue size distribution of the discrete-time Geo/G/1 queue with repeated customers," Queueing Systems, vol. 21, no. 1-2, pp. 199-215, 1995.

[7] I. Atencia and P. Moreno, "A discrete-time Geo/G/1 retrial queue with general retrial times," Queueing Systems, vol. 48, no. 1-2, pp. 5-21, 2004.

[8] J. R. Artalejo and Q.-L. Li, "Performance analysis of a blockstructured discrete-time retrial queue with state-dependent arrivals," Discrete Event Dynamic Systems, vol. 20, no. 3, pp. 325347, 2010.

[9] R. Nobel and P. Moreno, "A discrete-time retrial queueing model with one server," European Journal of Operational Research, vol. 189, no. 3, pp. 1088-1103, 2008.

[10] J. R. Artalejo and V. Pla, "On the impact of customer balking, impatience and retrials in telecommunication systems," Computers and Mathematics with Applications, vol. 57, no. 2, pp. 217229, 2009.

[11] A. Aboul-Hassan, S. I. Rabia, and F. A. Taboly, "A discrete time $\mathrm{Geo} / \mathrm{G} / 1$ retrial queue with general retrial times and balking customers," Journal of the Korean Statistical Society, vol. 37, no. 4, pp. 335-348, 2008.

[12] J. Wang and P.-F. Zhou, "A batch arrival retrial queue with starting failures, feedback and admission control," Journal of Systems Science and Systems Engineering, vol. 19, no. 3, pp. 306-320, 2010.

[13] J. R. Artalejo and G. Choudhury, "Steady state analysis of an $M / G / 1$ queue with repeated attempts and two-phase service," Quality Technology \& Quantitative Management, vol. 1, no. 2, pp. 189-199, 2004.

[14] I. Atencia and P. Moreno, "A discrete-time retrial queue with 2nd optional service," in Proceeding of the 5th International Workshop on Retrial Queues, pp. 117-121, 2004.

[15] G. Choudhury and K. Deka, "An $M^{X} / G / 1$ unreliable retrial queue with two phases of service and Bernoulli admission mechanism," Applied Mathematics and Computation, vol. 215, no. 3, pp. 936-949, 2009.

[16] V. G. Kulkarni and B. D. Choi, "Retrial queues with server subject to breakdowns and repairs," Queueing Systems, vol. 7, no. 2, pp. 191-208, 1990.

[17] J. Wang, J. Cao, and Q. Li, "Reliability analysis of the retrial queue with server breakdowns and repairs," Queueing Systems, vol. 38, no. 4, pp. 363-380, 2001.

[18] J. Wang, B. Liu, and J. Li, "Transient analysis of an $M / G / 1$ retrial queue subject to disasters and server failures," European Journal of Operational Research, vol. 189, no. 3, pp. 1118-1132, 2008.

[19] Q.-L. Li, Y. Ying, and Y. Q. Zhao, "A $B M A P / G / 1$ retrial queue with a server subject to breakdowns and repairs," Annals of Operations Research, vol. 141, pp. 233-270, 2006.

[20] I. Atencia and P. Moreno, "A discrete-time Geo/G/1 retrial queue with the server subject to starting failures," Annals of Operations Research, vol. 141, pp. 85-107, 2006.
[21] J. Wang and Q. Zhao, "Discrete-time Geo/G/1 retrial queue with general retrial times and starting failures," Mathematical and Computer Modelling, vol. 45, no. 7-8, pp. 853-863, 2007.

[22] I. Atencia, I. Fortes, and S. Sánchez, "A discrete-time retrial queueing system with starting failures, Bernoulli feedback and general retrial times," Computers and Industrial Engineering, vol. 57, no. 4, pp. 1291-1299, 2009.

[23] J. Wang and P. Zhang, "A discrete-time retrial queue with negative customers and unreliable server," Computers and Industrial Engineering, vol. 56, no. 4, pp. 1216-1222, 2009.

[24] I. Atencia and P. Moreno, "A discrete-time Geo/G/1 retrial queue with server breakdowns," Asia-Pacific Journal of Operational Research, vol. 23, no. 2, pp. 247-271, 2006.

[25] S. W. Fuhrmann and R. B. Cooper, "Stochastic decompositions in the $M / G / 1$ queue with generalized vacations," Operations Research, vol. 33, no. 5, pp. 1117-1129, 1985.

[26] J. R. Artalejo and J. I. Falin, "Stochastic decomposition for retrial queues," Top, vol. 2, no. 2, pp. 329-342, 1994. 


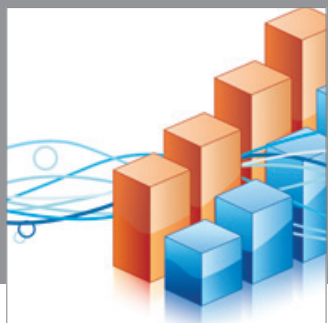

Advances in

Operations Research

mansans

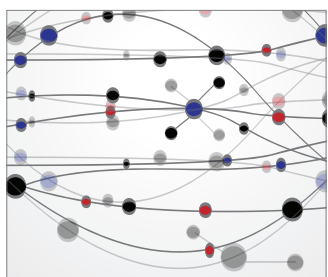

The Scientific World Journal
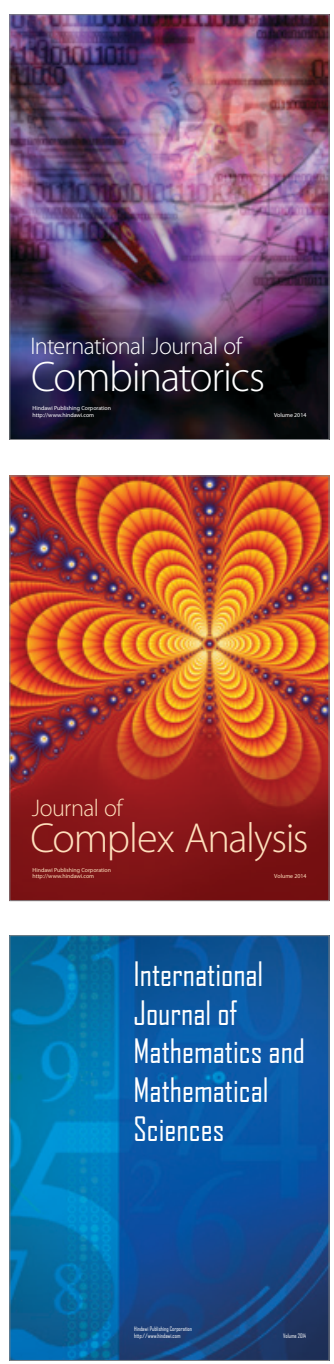
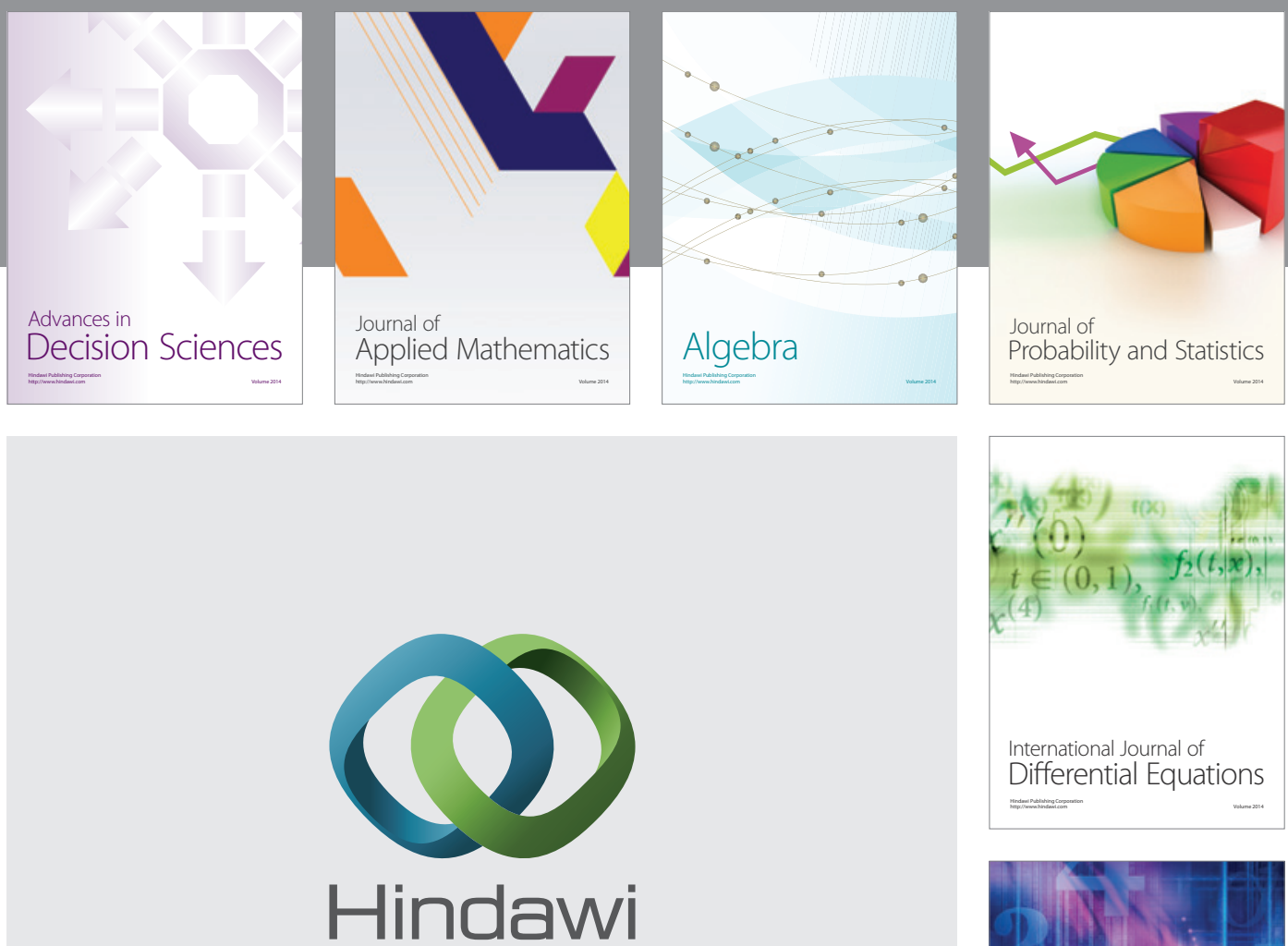

Submit your manuscripts at http://www.hindawi.com
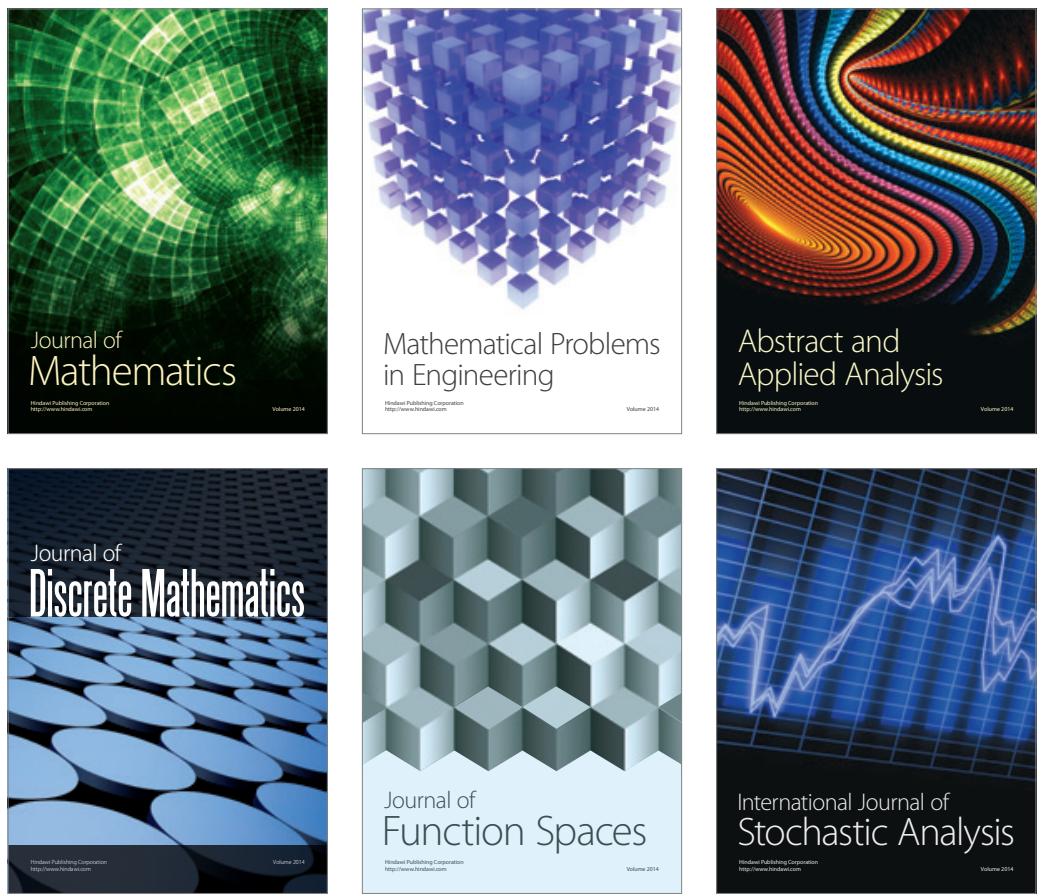

Journal of

Function Spaces

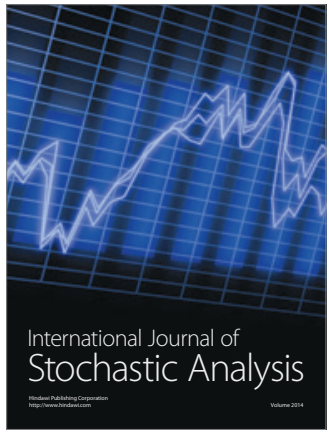

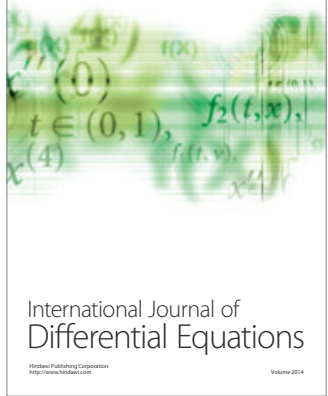
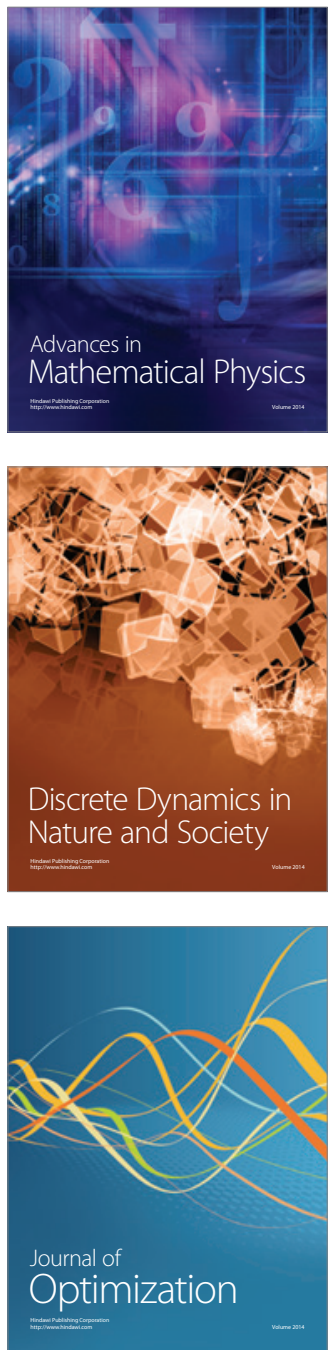